OPEN ACCESS

Edited by:

Heriberto Fernandez, Austral University of Chile, Chile

Reviewed by: Dalia Denapaite, University of Trento, Italy María Teresa Ulloa, University of Chile, Chile

${ }^{*}$ Correspondence: Margarita Camorlinga-Ponce margaritacamorlinga@yahoo.com Javier Torres uimeip@gmail.com

${ }^{\dagger}$ These authors have contributed equally to this work

Specialty section: This article was submitted to

Clinical Microbiology,

a section of the journal Frontiers in Cellular and Infection Microbiology

Received: 28 February 2020 Accepted: 30 December 2020 Published: 11 February 2021

Citation:

Camorlinga-Ponce $M$ Gómez-Delgado A, Aguilar-Zamora $E$, Torres RC, Giono-Cerezo S, Escobar-Ogaz A and Torres $J$ (2021) Phenotypic and Genotypic Antibiotic Resistance Patterns in Helicobacter pylori Strains From Ethnically Diverse Population in México. Front. Cell. Infect. Microbiol. 10:539115. do: $10.3389 /$ fcimb.2020.539115

\section{Phenotypic and Genotypic Antibiotic Resistance Patterns in Helicobacter pylori Strains From Ethnically Diverse Population in México}

\author{
Margarita Camorlinga-Ponce ${ }^{1 *}$, Alejandro Gómez-Delgado ${ }^{1}$, \\ Emmanuel Aguilar-Zamora ${ }^{1,2}$, Roberto C. Torres ${ }^{1}$, Silvia Giono-Cerezo ${ }^{3}$, \\ Antonio Escobar-Ogaz ${ }^{1}$ and Javier Torres ${ }^{1 * t}$ \\ 1 Unidad de Investigación en Enfermedades Infecciosas, UMAE Pediatría, Instituto Mexicano del Seguro Social, Ciudad de \\ México, Mexico, 2 Posgrado en Biomedicina y Biotecnología Molecular, Escuela Nacional de Ciencias Biológicas, Instituto \\ Politécnico Nacional, Ciudad de México, Mexico, ${ }^{3}$ Departamento de Microbiología, Escuela Nacional de Ciencias Biológicas, \\ Instituto Politécnico Nacional, Ciudad de México, Mexico
}

Helicobacter pylori strains carry a range of mutations in genes that confer antimicrobial resistance and restrict the available options to treat the infection. Latin America is a region that conserve a large number of indigenous communities relatively isolated that practice a traditional medicine without consumption of drugs. We hypothesized that rates of antibiotic resistance are lower in these communities. Recent progress in whole-genome sequencing has allowed the study of drug susceptibility by searching for the known mutations associated with antibiotic resistance. The aim of this work was to study trends of antibiotic resistance over a 20-year period in Mexican $\mathrm{H}$. pylori strains and to compare susceptibility between strains from Mexican mestizos and from indigenous population; we also aimed to learn the prevalence of mutational patterns in genes gyrA, gyrB, $r d x A$, frxA, rpsU, omp11, $d p p A$, and 23S rRNA and its association with phenotypic tests. Resistance to clarithromycin, metronidazole, amoxicillin and levofloxacin was determined in167 $\mathrm{H}$. pylori isolates by E-test, and the occurrence of mutational patterns in specific genes was determined by whole genome sequencing (WGS). The trend of resistance over 20 years in mestizo isolates showed significant resistant increase for clarithromycin and levofloxacin to frequencies that banned its clinical use. Resistance in $H$. pylori isolates of native communities was lower for all antibiotics tested. Phenotypic resistance showed good to moderate correlation with genotypic tests. Genetic methods for characterizing antibiotic resistance require further validation in each population.

Keywords: Helicobacter pylori, antibiotic resistance, whole genome sequencing, phenotype, genotype, indigenous communities 


\section{INTRODUCTION}

Drug resistance is recognized as one of the major threats to worldwide public health (Laxminarayan et al., 2013). The problem is a highly pressing issue because of the rapid spread of multi-drug resistant bacteria, some of which are no longer treatable with the available antibiotics (Dadgostar, 2019). Antibiotic resistance is the main risk factor for failure to respond to treatment of $H$. pylori infection (Alfizah et al., 2014). H. pylori is an ancient microorganism that has coevolved with man for at least 60,000 years (Moodley et al., 2012); still, it represents a significant public health issue since it is the main risk factor for gastric cancer, which is the third cause of death because of all cancers (Hooi et al., 2017). It is estimated that over $90 \%$ of all cases of stomach cancer are attributed to H. pylori (Moss, 2017). Eradication treatment against $H$. pylori may halt the progression of chronic atrophic gastritis and intestinal metaplasia, preventing the development of gastric cancer (Mera et al., 2005; Fuccio et al., 2009).

H. pylori is a small, curved, motile, Gram-negative bacillus that colonizes the stomach mucosa of more than $50 \%$ of the world population, particularly in developing countries of Africa, Latin America, the Caribbean and Asia (Sjomina et al., 2018). In contrast, the lowest prevalence is reported in Northern America and Oceania, whereas it is declining in Japan (Leja et al., 2016).

Although there is no global recommendation on the regimen for eradication of $H$. pylori, there are regional consensus (e.g., European, Latin American, Asiatic) (Rollan et al., 2014; Malfertheiner et al., 2017; Sheu et al., 2017) that in the last decades have recommended mostly a "standard" triple therapy (proton pump inhibitor + clarithromycin + amoxicillin) as the first choice of treatment. However, during the last years, most of these regions have reconsidered their recommendations, because of the increase in resistance to clarithromycin (Thung et al., 2016). Current recommendations vary among regions because susceptibility to drugs is different in each geographic area, but also because of other factors like availability and cost of the drugs. The most recently recommended drugs for $H$. pylori eradication include fluoroquinolones and rifamycin (Goderska et al., 2018).

The H. pylori strains may carry a range of mutations in genes that confer antimicrobial resistance and limit the available options to treat $H$. pylori infection. Resistance to clarithromycin is commonly associated to three point mutations in the $23 \mathrm{~S}$ ribosomal RNA (rRNA) gene, A2143G (69.8\%), A2142G (11.7\%), and A2142C (2.6\%) (Thung et al., 2016); whereas other less frequent mutations include A2115G, G2141A, A2144T, and T2289C. Mutations C2694A and T2717C have been associated with low resistance levels (Marques et al., 2020). Other point mutations have been described in the literature (A1821G, G1826A, and T1830C, C2245T, G2224A, but their role in clarithromycin resistance requires confirmation (Rimbara et al., 2007; Alarcón-Millán et al., 2016; Hashemi et al., 2019). These mutations prevent the interaction of the macrolide with the $23 \mathrm{~S}$ rRNA. The prevalence of $H$. pylori resistance to clarithromycin varies among regions. Reported resistance in West/central and South Europe is $>20 \%$, and in North Europe it is $<10 \%$ (Megraud et al., 2013); whereas in strains from Latin American countries the overall prevalence of resistance is $12 \%$, although frequencies ranged from $0 \%$ to $60 \%$ (Camargo et al., 2015).

Reported $H$. pylori resistance to metronidazole is $33.1 \%$ in Europe and $40 \%$ in the USA, whereas higher rates have been reported in developing countries, with frequencies as high as $80 \%$ (Rollan et al., 2014; Thung et al., 2016); still, metronidazole is frequently used in eradication regimens (Fiorini et al., 2018). Mutations in the $r d x A$ gene have been identified as the main cause of resistance to this drug (Goodwin et al., 1998). Although it has been found that mutations in other genes could also influence resistance to metronidazole, including $f r x A, m d a B, o m p 11, d d p A$ or $r p s U$ genes, and genes involved in transcription regulation of $r d x A$ or in overexpression of $h f A$ efflux pump; although additional studies are required to clearly demonstrate their association with metronidazole resistance (Alba et al., 2017; Saranathan et al., 2020). H. pylori resistance to amoxicillin has remained low worldwide, with reported frequencies of $<2 \%$ in European countries (Thung et al., 2016) and 6.6\% in Bangladesh (Nishizawa and Suzuki, 2014). Amoxicillin binds to penicillin binding proteins (PBP) interfering with peptidoglycan synthesis causing lysis of replicating bacteria and resistance is mainly associated with mutations in the $p b p$ genes (Nishizawa and Suzuki, 2014). Resistance to fluoroquinolones has been determined in a few countries, with rates of $3.3 \%$ in France, $34.5 \%$ in China, $31.9 \%$ in the USA (Thung et al., 2016) and $40 \%$ in Venezuela (Lopez Gasca et al., 2018). Levofloxacin is a $3^{\text {nd }}$ generation fluoroquinolone currently suggested for H. pylori eradication (Malfertheiner et al., 2017); it inhibits DNA gyrase and topoisomerase and mutations in the gyrA gene are associated with resistance (Nishizawa and Suzuki, 2014).

Rates of antibiotic resistance may also vary within countries, where differences in drug exposure may be observed within communities. Latin America is a region that conserve a large number of indigenous communities that have remained relatively isolated and often practice a traditional medicine without consumption of drugs. We hypothesized that rates of antibiotic resistance are lower within these communities. In Mexico, approximately 7\% of the Mexican population (over 7 million Mexicans) speak a native language. These ethnic groups have different economic development and lifestyles with little access to medical services (Romero-Hidalgo et al., 2017).

Recent technical progress in whole-genome sequencing has allowed the study of drug susceptibility by searching for the known mutations associated with resistance; this approach may led to the discovery of novel mutations responsible for antibiotic resistance. The aim of this work was to compare the susceptibility to antibiotics in $H$. pylori strains isolated in a mestizo community versus isolates from indigenous populations. We also aimed to evaluate the performance of resistance detection by genome sequencing as compared with the phenotypic detection, measuring susceptibility in agar plates. 


\section{MATERIALS AND METHODS}

\section{Bacterial Strains}

A total of $167 \mathrm{H}$. pylori strains isolated during the period from 1997 to 2017 were studied, 111 strains were isolated from biopsies of Mexican mestizo adults with non-atrophic gastritis, intestinal metaplasia, gastric cancer, and duodenal ulcer. Biopsy specimens were cultured as previously described (Avilés-Jiménez et al., 2012). All patients were seen at the Centro Médico Nacional Siglo XXI, Instituto Mexicano del Seguro Social in Mexico City. The study was approved by the ethical committee from the Instituto Mexicano del Seguro Social. In addition, $56 \mathrm{H}$. pylori strains previously isolated from Mexican indigenous people were included (Camorlinga-Ponce et al., 2011). All $H$. pylori organisms were stored at $-70^{\circ} \mathrm{C}$ until tested.

\section{Phenotypic Characterization of Antimicrobial Susceptibility}

The antimicrobial susceptibility of $H$. pylori to amoxicillin, clarithromycin, levofloxacin, and metronidazole was determined using the Epsilometer test (E test) (Torres et al., 2001), a test that whereas is not considered as the reference methods, it is commonly used for a number of antibiotics in different bacteria. A recent study reported a good agreement for levofloxacin, clarithromycin, and metronidazole when compared with the agar dilution method, considered as the gold standard (Miftahussurur et al., 2020). H. pylori isolates were grown for 2 days on Columbia blood agar plates (Becton Dickinson, New Jersey, USA) and growth suspended in Columbia broth to achieve a McFarland opacity of 3. Bacterial suspension was spread on Muller Hinton agar medium with 5\% of sheep blood (Becton Dickinson, New Jersey, USA) and the E-test strips (Liofilchem, Roseto degli Abruzzi, Italy) were placed on the plate and incubated at $37^{\circ} \mathrm{C}$ for $72 \mathrm{~h}$ with a $10 \% \mathrm{CO}_{2}$ atmosphere (Nuaire, Plymouth, Minn). The MIC was defined by the point of intersection of the inhibitory zone with the strip. MIC values were defined according to the clinical breakpoints proposed in the sixth version of the EUCAST (European Committee on Antimicrobial Susceptibility Testing, EUCAST, $9^{\text {th }}$ version, 2019) for $H$. pylori. The cutoff values for resistance were $>8 \mathrm{mg} / \mathrm{L}$ for metronidazole, $>0.125 \mathrm{mg} / \mathrm{L}$ for amoxicillin, $>1$ $\mathrm{mg} / \mathrm{L}$ for levofloxacin, and $>0.5 \mathrm{mg} / \mathrm{L}$ for clarithromycin. Two reference strains were used as controls, H. pylori ATCC 43504 with MICs for clarithromycin, amoxicillin, metronidazole, and levofloxacin of $0.016,0.016,64$, and $0.064 \mathrm{mg} / \mathrm{L}$, respectively and strain $H$. pylori 26695 with MICs for clarithromycin, amoxicillin, metronidazole, and levofloxacin of $0.98,0.94,0.75$, and 0.32 $\mathrm{mg} / \mathrm{L}$, respectively.

\section{DNA Extraction and Sequencing}

Genomic DNA was extracted from $93 \mathrm{H}$. pylori strains using the DNeasy Mini kit (Qiagen, Hilden, Germany) according to the manufacturer's instructions. Nine isolates were sequenced with the HiSeq 2000 platform (Illumina, San Diego, CA, USA) using the paired-end method, as previously described (MuñozRamírez et al., 2017). The remaining isolates were sequenced at the Weimer laboratory at the University of California, Davis,
(USA) within the $100 \mathrm{~K}$ Pathogen Genome Project (Weimer, 2017). Isolates (65) were sequenced using Illumina HiSeq2500 (Weis et al., 2016; Draper et al., 2017). While 19 were done using Pacific Biosciences as previously described (Kong et al., 2014). PacBio reads were processed with the SMRT Analysis package (version 1.3). Genomes were assembled de novo by using the hierarchical genome assembly process (HGAP) and polished using Quiver to obtain final consensus assemblies. For the Illumina paired-end 150 reads, we tested k-mers lengths from 31 to 121 using the VelvetOptimiser script (version 2.2.4) (Zerbino and Birney, 2008). The k-mer length that produced the best assembly according to the N50 contig was used to generate the final assemblies using ABySS (version 2.0.2) (Simpson et al., 2009) (Supplementary Table 1).

\section{Identification of Mutations in Resistance-Associated Genes}

Genomic variations were identified in genes conferring antibiotic resistance: $23 \mathrm{~S}$ rRNA for clarithromycin; $r d x A$, frxA, rps $U$, omp11, and $d p p A$ for metronidazole; pbp1, pbp2, pbp3 for amoxicillin; and gyrA and $g y r B$ for levofloxacin. First, open reading frames (ORFs) were predicted from de novo assemblies by a genomic annotation using the Prokka software v1.12 (Seemann, 2014) and the proteome of the 26695 strain (NC_000915) as primary source. Then, genomic coordinates of ORFs were used to extract the entire sequence of target genes. Sequences were then aligned according to genes of the 26695 reference strain (GeneBank accession number U27270.1) using muscle v3.8.31 (Edgar, 2004) and SNPs were called from alignments using SNIPPY (v.4.6.0) (Page et al., 2016) (https:// github.com/tseemann/snippy) following the parameters: minimum coverage $20 \mathrm{X}$, a minimum base quality score of 30 and a proportion for variant evidence of 0.9 . Mutations were considered according to SNIPPY outputs and visualized using Artemis v18.1.0 (Carver et al., 2012) or MEGAS's sequence editor (Tamura et al., 2013).

\section{Statistical Analysis}

The sociodemographic variables were analyzed as follows, gender and clinical diagnosis were compared with the Kruskal-Wallis test. To compare frequency of resistance to antibiotics between the different periods of isolation the $\mathrm{X}^{2}$ linear trend was used. Frequency of resistance to one or more antibiotics among the study populations was analyzed by means of $\mathrm{X}^{2}$; ORs (Odds Ratio) and their $95 \%$ confidence intervals were also estimated. In all cases, a p-value $<0.05$ was considered as statistically significant.

\section{RESULTS}

\section{Characteristics of the H. pylori Strains Studied}

Table 1 describes the characteristics of the patients from whom H. pylori strains were isolated. A total of $167 \mathrm{H}$. pylori strains were included in this study, of these, 111 were isolated from Mexican mestizos (mean age, $48.7 \pm 14.2$ years; 34 males and 77 
TABLE 1 | Characteristics of patients from whom $\mathrm{H}$. pylori was isolated in Mexico during 1997-2017.

\begin{tabular}{lccc}
\hline Time Period & $\begin{array}{c}\text { Total } \\
\mathbf{1 9 9 7 - 2 0 1 7}\end{array}$ & $\begin{array}{c}\text { Mestizo population } \\
\mathbf{1 9 9 7 - 2 0 1 7}\end{array}$ & $\begin{array}{c}\text { Native community } \\
\mathbf{2 0 0 2 - 2 0 0 4}\end{array}$ \\
\hline $\begin{array}{l}\text { No of strains } \\
{ }^{*} \text { Age }\end{array}$ & $\mathrm{n}=167$ & $\mathrm{n}=111$ & $\mathrm{n}=56$ \\
Age, mean(SD) & $50.72(14.27)$ & $48.7(14.2)$ & $41.63(20.03)$ \\
${ }^{* *}$ Gender & & & \\
Male, $\mathrm{n}(\%)$ & $48(28.7)$ & $34(30.6)$ & $21(37.5)$ \\
Female, $\mathrm{n}(\%)$ & $119(71.2)$ & $77(69.4)$ & $35(62.5)$ \\
Diagnosis & 119 & 63 & 56 \\
Gastritis & 18 & 18 & 0 \\
$\begin{array}{l}\text { Pre-neoplastic } \\
\text { Lesions }\end{array}$ & & & 0 \\
Gastric cancer & 23 & 23 & 0 \\
Duodenal ulcer & 7 & 7 & \\
\hline
\end{tabular}

${ }^{*} t=-3.03 ; p=0.003$.

${ }^{*} X^{2}=0.795 ; p=0.37$ OR $=0.74$ I. C. $95 \%=(0.38-1.45)$.

females). The clinical diagnosis in these patients were chronic gastritis, pre-neoplastic lesions, gastric cancer and duodenal ulcer. $56 \mathrm{H}$. pylori strains from Mexican indigenous people were also studied, all individuals with chronic gastritis (mean age $41.63 \pm 20.0$ years; 21 males and 35 females).

\section{Antimicrobial Susceptibility}

Analysis of the antimicrobial susceptibility of the 167 isolates reveled high resistance for metronidazole (58.6\%), and moderate to levofloxacin (18.5\%) and clarithromycin (8.9\%), while only $1.8 \%$ were resistant to amoxicillin. We next analyzed the trend of resistance over a 20-year period for the mestizo isolates (Figure 1 and Table 2) and found a constant increase from 1997 to 2017 for clarithromycin $(1.85 \%-32.2 \%, \mathrm{p}=0.000)$ and levofloxacin $(9.2 \%-58.1 \%, \mathrm{p}=0.000)$. In contrast, resistance to metronidazole tend to decrease $(73 \%-51.6 \%)$; whereas for amoxicillin resistance was not detected during 1997-2011 but was already present in $6.5 \%$ of the 2017 isolates.

Compared with the mestizo strains, resistance among the isolates of native communities was lower for metronidazole (39.3\%), very low for clarithromycin and amoxicillin (1.8\% for both) and absent for levofloxacin (0\%) (Table 2). Among all the $167 \mathrm{H}$. pylori strains tested, $30.5 \%$ were susceptible to all four antibiotics tested, which corresponded to $15.3 \%$ of the mestizos and $66 \%$ of the indigenous communities (Table 3). Among all studied strains $56 \%$ were resistant to at least one antibiotic and

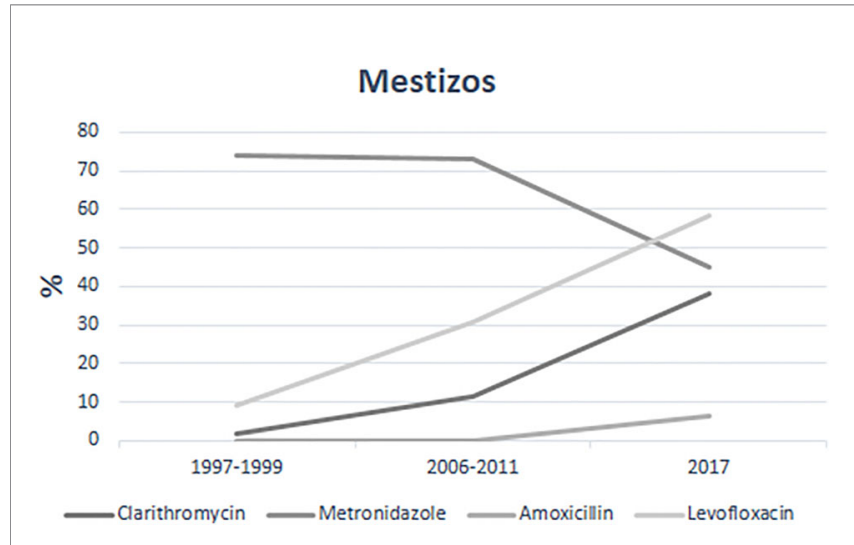

FIGURE 1 | Trends of Antimicrobial resistance in $H$. pylori strains isolated from Mexican mestizos during the period 1997-2017.

interestingly, $65 \%$ of the mestizo vs. $37 \%$ of the indigenous strains presented this pattern. Finally, multidrug resistance was also higher in mestizo (18.9\%) than in indigenous strains (1.7\%) (Table 3). Among the multi-resistant mestizo strains, dual resistance to metronidazole/levofloxacin was more frequently observed $(16.2 \%)$, followed by resistance to clarithromycin/metronidazole/levofloxacin in $4.5 \%$ of the tested strains. Resistance to both metronidazole/levofloxacin rose from $7.4 \%$ in 1997 to 1999 to $32 \%$ in 2017. A high resistance rate of $25.8 \%$ was detected for clarithromycin/ metronidazole among $H$. pylori isolates from mestizos. No strain was resistant to the four antibiotics tested. Of note, among the strains of indigenous origin, only one isolate presented double resistance (clarithromycin/metronidazole).

\section{Mutation Analysis in the Resistance Genes}

Of the 167 strains included in the study, 93 strains (74 mestizos and 19 indigenous) were whole-genome sequenced, 84 in this study and 9 reported previously (Muñoz-Ramírez et al., 2017) (Supplementary Table 1). Seven of these 93 strains were phenotypically resistance to clarithromycin; of these, one presented the A2146G (formerly A2142G) amino acid variant and four presented the variant A2147G (formerly A2143G) (Table 4); whereas among the 86 sensitive strains two presented variants reported associated with resistance. In this analysis we checked for mutations in the two copies of the $23 \mathrm{~S}$

TABLE 2 | Antimicrobial resistance of H. pylori strains isolated from Mexican mestizos over a period of 20 years and from native populations.

\begin{tabular}{|c|c|c|c|c|}
\hline Years & ${ }^{\mathrm{a} C l a r i t h r o m y c i n ~ N o}(\%)$ & ${ }^{\mathrm{b}}$ Metronidazole No (\%) & Amoxicillin No (\%) & ${ }^{c}$ Levofloxacin No (\%) \\
\hline $1997-1999(n=54)$ & $1(1.85)$ & $41(75.9)$ & 0.0 & $5(9.2)$ \\
\hline $2017(n=31)$ & $10(32.2)$ & $16(51.6)$ & $2(6.5)$ & $18(58.1)$ \\
\hline \multicolumn{5}{|l|}{ Native community } \\
\hline $2002-2004(n=56)$ & *1 (1.8) & **22 (39.3) & $* * \star 1(1.8)$ & 0.0 \\
\hline Total $\mathrm{N}=167$ & $15(8.9)$ & $98(58.6)$ & $3(1.8)$ & $31(18.5)$ \\
\hline
\end{tabular}

${ }^{a} X^{2}$ for linear trend $=15.87, p=0.0007 ;{ }^{b} X^{2}$ for linear trend $=4.89, p=0.026 ;{ }^{c} X^{2}$ for linear trend $=23.15 ; p=0.0000$.

${ }^{*} X^{2}=5.33, p=0.02, O R=7.93(1.01-62.0)$ mestizo vs native populations; ${ }^{*} X^{2}=13.07, p=0.0002, O R=3.35(1.72-6.55)$ mestizo vs native populations; ${ }^{* * *}$ Fisher Exact $=0.7$,

$O R=1.00(0.89-11.37)$ mestizo vs native populations. 
TABLE 3 | Patterns of antibiotic sensitivity of $H$. pylori strains isolated from indigenous and mestizo communities in Mexico.

Ethnic origin of $\boldsymbol{H}$. pylori strains

\begin{tabular}{|c|c|c|c|c|}
\hline Resistance & Indigenous n=56 (\%) & Mestizo $n=111$ (\%) & All $n=167(\%)$ & OR $(95 \% \mathrm{Cl})$ \\
\hline Resistance to one antibiotic & $21(37.5)$ & $73(65.7)$ & $94(56.3)$ & $0.31(0.16-0.61)$ \\
\hline
\end{tabular}

OR, Odds Ratio.

TABLE 4 | H. pylori mutations in genes associated with phenotype resistant to Clarithromycin, Levofloxacin, and Metronidazole.

\begin{tabular}{|c|c|c|}
\hline & Resistant No (\%) & Sensitive No (\%) \\
\hline Clarithromycin & $n=10$ & $n=100$ \\
\hline \multicolumn{3}{|l|}{ 23S rRNA } \\
\hline A2142G & $3(30)$ & 0 \\
\hline A2143G & 0 & $1(1)$ \\
\hline A2146G & $2(20)$ & $1(1)$ \\
\hline A2147G & $2(20)$ & $1(1)$ \\
\hline Levofloxacin & $\mathrm{n}=32$ & $\mathrm{n}=78$ \\
\hline \multicolumn{3}{|l|}{ GyrA } \\
\hline N87T & $4(12.5)$ & $7(8.9)$ \\
\hline N87K & $6(18.8)$ & $3(3.8)$ \\
\hline N87I & $8(25.0)$ & $5(6.4)$ \\
\hline T621 & 0 & $1(1.2)$ \\
\hline D91G & $1(3.1)$ & $1(1.2)$ \\
\hline D91Y & $1(3.1)$ & $2(2.5)$ \\
\hline N87T-D91G & $1(3.1)$ & 0 \\
\hline D91N & $1(3.1)$ & $4(5.1)$ \\
\hline \multicolumn{3}{|l|}{ GyrB } \\
\hline D481E & 0 & $2(2.5)$ \\
\hline \multirow[t]{2}{*}{ D481G } & 0 & $1(1.2)$ \\
\hline & & 0 \\
\hline Metronidazole & $\mathrm{n}=78$ & $\mathrm{n}=32$ \\
\hline \multicolumn{3}{|l|}{ rpsU } \\
\hline $\mathrm{A} 11 \mathrm{~T}$ & $3(3.8)$ & 0 \\
\hline G9S & $3(3.8)$ & 0 \\
\hline $\mathrm{R} 36 \mathrm{G}$ & 0 & $3(9.3)$ \\
\hline \multicolumn{3}{|l|}{ omp11 } \\
\hline V148I & 12(15.3) & 12(37.5) \\
\hline T13A & $24(30.7)$ & $31(96.8)$ \\
\hline
\end{tabular}

rRNA operon present in $H$. pylori. For levofloxacin, among the 93 strains 22 were resistant and 17 presented amino acid variants in position 87 and 91 of GyrA (Table 4); however, 14 of the 71 sensitive also presented these variants. The most common variant found in GyrA was N87I, whereas amino acid variants in GyrB were in two resistant strains but present in four of the 71 sensitive isolates.

Regarding metronidazole, there are no reports on mutations clearly associated with resistance, accordingly, we describe all the observed mutations. Mutations in $r p s U$ leading to variants A11T and G9S were found in resistant strains (Table 4); the A11T mutants had a MIC $>256$ to metronidazole. Two isolates sensitive to metronidazole presented the mutation $\mathrm{R} 36 \mathrm{G}$ in $r p s U$. In addition, we detected mutations in omp11, but the significance of this finding is unclear because mutations were found in both, strains resistant and sensitive to metronidazole (Table 4). In gene $d p p A$ mutations in I485L, Q382E, and $37 \mathrm{del}$ were present only in strains resistant to metronidazole.

A considerable number of mutations was found in $r d x A$ and amino acid variants were as much as 64 (Table 5), 32 present only in resistant strains, 22 present in both resistant and sensitive and 10 present only in sensitive strains. Besides the 64 nonsynonymous mutations in $r d x A$ we also found six frameshifts, three nonsense mutations, 32 missense mutations, and stop codons were observed in resistant isolates in positions 13,26 , 37, 72, 73, and 76. Similarly, in FrxA we found no amino acid variants clearly associated with metronidazole resistance, amino acid variants were present only in three resistant $H$. pylori strains,

TABLE 5 | Amino acid variants present in RdxA of H. pylori strains resistant or sensitive to metronidazole.

33 amino acid variants present only in resistant $H$. pylori strains

10 amino acid variants present only in sensitive $H$. pylori strains

22 amino acid variants present in both sensitive and resistant $H$. pylori strains
K2R, Q11X, R14K, H17Y, H25R, E27T, 30SR, A37V, S45N, Q50R, M56V, T58I, L62S, K63Q, S81L, P106D, P106S, V111A, P115R, A118S, C140R,C140G, S158M, G163D, G163V,K168R, A193V, K168R, A193V, E194G, A206T, I207T

H53R, A69Y, P96L, V123T, S158G, R176H, D205A, A206E, I207E, T208L

Q11H, R16G, R16H, T31E, T32A, M56I, D59N, A67V, R90K, H97Y, H97T, G98S, A118T, S128G, R131K, G170S, V172I, E175Q, A183V, C184R, Q197K, V204I.

TABLE 6 | Amino acid variants present in FrxA of $H$. pylori strains resistant or sensitive to metronidazole.

3 amino acid variants present only in resistant $H$. pylori strains

13 amino acid variants present only in sensitive $H$. pylori strains

14 amino acid variants present in both sensitive and resistant $H$. pylori strains
L52I, A85V, E169A

V7I, A16T, A32V, , I44V, R58G, K60R, V81L, D91N, N110D, N124S, A152V, E169A, E176K

L33M, A67T, A70G, L71I; F72S, T110S, N110H, F114Y, M125F, N129T, S130D, E176K, N182D, C193S 
14 were present in both sensitive and resistant strains and 13 present only in sensitive strains (Table 6).

\section{Evaluation of the Performance of Genetic Tests to Detect Antibiotic Resistance}

To measure the efficacy of molecular detection of resistance, we estimated the concordance of phenotype vs. genotype using the reported antibiotic resistance associated mutations for clarithromycin and levofloxacin and all found mutations for metronidazole (Table 7). Performance for clarithromycin had a substantial agreement $(\mathrm{K}=0.69)$, which is as good as in reports from other populations (Farzi et al., 2019; Tshibangu-Kabamba et al., 2020); we found two resistant strains with no mutation in 23S, suggesting that in our population other mechanisms are responsible for this phenotype. Thus, $23 \mathrm{~S}$ genotyping might be recommended for clarithromycin resistance in $H$. pylori strains from our community. Regarding levofloxacin, genotyping of $g y r A$ and gyrB had a moderate agreement $(K=0.49)$. Previous studies identified amino acid variants in positions 87 and 91 of GyrA associated with high MIC values for levofloxacin (Lopez Gasca et al., 2018; Hanafiah et al., 2019; Tuan et al., 2019). Genotyping of gyrA might not be an alternative to determine levofloxacin resistance if phenotypic tests are not available. For metronidazole, $r d x A$ and $f r x A$ turned out to be highly variable genes with an unusual number of SNPs, and no single mutation clearly associated with resistance resulting in a slight agreement $(<0.2)$; genotyping of $r p s U, d p p A, r d x A$, or $f r x A$ is not an option to identify metronidazole resistant strains. Regarding amoxicillin, we found a reduced number of resistant strains and those strains presented no mutations in PBPs. Mutations in resistance-associated genes increased after the period 1997 to 1999 as observed in the periods 2002 to 2011 and 2017 (Table 8), particularly for $23 \mathrm{~S}$ rRNA, gyrA, and $d p p A$ genes.

\section{DISCUSSION}

In 2017, $H$. pylori was categorized by the WHO as a bacterium with high antibiotic resistance that poses an important risk to human health (Dang and Graham, 2017). H. pylori antibiotic resistance is the main factor affecting the success of current therapeutic regimens and alternatives for suitable antibiotics to treat this infection are becoming limited. Clarithromycin and amoxicillin are the antibiotics of the standard triple therapy, still in use in many regions for the of eradication H. pylori (Suzuki

TABLE 7 | Analysis of correlation between antibiotic resistance phenotype and genotype.

\begin{tabular}{lcccc}
\hline Antibiotic & Genotype & \multicolumn{2}{c}{ Phenotype } & Kappa \\
\cline { 3 - 4 } & & S & $\mathbf{R}$ & \\
\hline Clarithromycin & $\mathrm{S}$ & 97 & 3 & $0.67(0.85-0.91)$ \\
Levofloxacin & $\mathrm{R}$ & 3 & 3 & \\
Metronidazole & $\mathrm{S}$ & 53 & 1 & $0.5(0.66-0.71)$ \\
& $\mathrm{R}$ & 27 & 29 & \\
& $\mathrm{~S}$ & 32 & 27 & $0.29(0.47-0.52)$ \\
& $\mathrm{R}$ & 10 & 32 &
\end{tabular}

TABLE 8 | Mutational analysis in genes involved with antibiotics resistance of Mexican $H$. pylori strains isolated over a period of 20 years.

\begin{tabular}{|c|c|c|c|}
\hline & $1997-1999 n=39$ & $2002-2011 n=40$ & $2017 N=31$ \\
\hline \multicolumn{4}{|l|}{ 23S rRNA } \\
\hline \multicolumn{4}{|l|}{$\mathrm{n}=7$} \\
\hline A2146G & & $1(14.2)$ & $3(42.8)$ \\
\hline A2147G & & & $1(14.2)$ \\
\hline \multicolumn{4}{|l|}{ Levofloxacin } \\
\hline \multicolumn{4}{|l|}{$\mathrm{n}=22$} \\
\hline \multicolumn{4}{|l|}{ GyrA } \\
\hline N87T & 0 & $1(4.5)$ & 0 \\
\hline N87K & & $1(4.5)$ & $3(13.6)$ \\
\hline N87I & & $2(9)$ & $4(18.1)$ \\
\hline D91G & & & $1(4.5)$ \\
\hline D91Y & & & $1(4.5)$ \\
\hline N87T-D91G & & $1(4.5)$ & \\
\hline D91N & & $2(9)$ & $1(4.5)$ \\
\hline \multicolumn{4}{|l|}{ Metronidazole } \\
\hline \multicolumn{4}{|l|}{$n=59$} \\
\hline \multicolumn{4}{|l|}{ rpsU } \\
\hline $\mathrm{A} 11 \mathrm{~T}$ & $1(1.6)$ & & $1(1.6)$ \\
\hline G9S & & $3(5)$ & \\
\hline \multicolumn{4}{|l|}{ Omp11 } \\
\hline V148I & $5(8.4)$ & $4(6.7)$ & $2(3.3)$ \\
\hline $\mathrm{T} 13 \mathrm{~A}$ & $8(13.5)$ & $9(15.2)$ & $3(5)$ \\
\hline \multicolumn{4}{|l|}{$d p p A$} \\
\hline $37 \mathrm{del}$ & & $1(1.6)$ & $3(5)$ \\
\hline |485L & & $4(6.7)$ & \\
\hline $1485 \mathrm{~V}$ & & $3(5)$ & $4(6.7)$ \\
\hline $\mathrm{A} 212 \mathrm{E}$ & & 12(20.3) & $8(13.5)$ \\
\hline Q382E & & $3(5)$ & \\
\hline
\end{tabular}

et al., 2019). However, the resistance to these antimicrobials has increased rapidly during the last decade in several countries (Ogata et al., 2013; Miftahussurur et al., 2016; Park et al., 2016).

We aimed to study the pattern of antibiotic resistance in Mexican mestizo population over a period of 20 years. During this period, we found a significant increase in the rate of resistance to clarithromycin and levofloxacin and moderate to amoxicillin; while resistance to metronidazole decreased. Resistance to clarithromycin was low in the first year of the survey, but after 20 years, it increased to levels that banned its clinical use; similar findings have been reported in Taiwan (65\%) (Wu et al., 2015), Chile (31.2\%) (Gonzalez-Hormazabal et al., 2018) and Brazil (19.5\%) (Ogata et al., 2013). Based on our studies, the most recent Mexican consensus no longer recommends the standard triple therapy for H. pylori eradication (Bosques-Padilla et al., 2018).

Over the 20-year study period, the resistance to metronidazole decreased from $75 \%$ to $51.6 \%$, a significant reduction that might be related to the fact that its previous widespread use (to treat diarrhea, parasitic infections or gynecological disorders), which favored the selection of resistant strains, has been drastically reduced in the last decades, particularly after the act that commanded written prescription to get antibiotics (Vázquez Tsuji Óscar, 2010). The current $51 \%$ rate of resistance is similar to that reported in Alaska (44\%), but lower than China (56.6\%), Vietnam (69.9 and \%), and Colombia (83\%) (Ierardi et al., 2013; Camargo et al., 2015).

After failure of the standard triple therapy, the Maastricht IV guidelines recommended levofloxacin as a salvage 
therapy (Malfertheiner et al., 2017) and other countries even suggested levofloxacin as first line therapy (Heo and Jeon, 2014; Liou et al., 2016). We found that in Mexico, resistance to levofloxacin rise from $9 \%$ to $58 \%$ in a 20 -year period; a $10 \%$ resistance has been suggested as the threshold to use levofloxacin as empiric first line therapy (Thung et al., 2016). Levofloxacin was clinically useful at the beginning of the survey, but now it is useless to eradicate $H$. pylori infection in mestizo population. This work is the first report on levofloxacin resistance in $H$. pylori in Mexico, but several studies have reported high levofloxacin resistance in Europe and Asia (Megraud et al., 2013; Alfizah et al., 2014; Smith et al., 2014; O’Connor et al., 2015). In Mexico a recent clinical trial compared the utility of triple therapy with clarithromycin versus levofloxacin and found a poor efficacy with both treatments with eradication rates close to $60 \%$ (Ladrón-de-Guevara et al., 2019). Quinolones have been widely used to treat urinary tract infections and other infections in Mexico, and the observed high resistance in H. pylori strains is most probably the result of exposure when other infections are treated. Studies reporting antibiotic resistance over a period of years are scarce in Latin America, our work clearly show a sharp increase in resistance to clarithromycin and levofloxacin and also the worrying appearance of resistance to amoxicillin. These pronounced changes in susceptibility are an indication of the need to monitor patterns of resistance, particularly in countries with high prevalence of the infection, including the Latin American region (Curado et al., 2019). The resistance rates to clarithromycin, metronidazole, and levofloxacin have increased over time globally, which agrees with studies presenting evidence of an association between antibiotic consumption and antibiotic resistant in $H$. pylori infection (Megraud et al., 2013; Camargo et al., 2015). In Mexico clarithromycin, amoxicillin and metronidazole are antibiotics commonly used to treat other infections, which would also favor this increase in resistance. Therapies including levofloxacin are currently recommended; however, in H. pylori isolates from 2017, we found levofloxacin resistance in $58 \%$ and multiresistance to metronidazole and levofloxacin of $32 \%$, discarding their clinical use for this infection.

A second aim of this study was to contrast the antimicrobial susceptibility to the most commonly used antibiotics in Mexican $H$. pylori strains isolated from two ethnically different groups. We found that $H$. pylori strains from mestizo population presented higher drug resistance than isolates from native communities. Native communities in Mexico usually follow its cultural customs including traditional medicine where the use of antibiotics is limited (Guzman-Rosas, 2016). It is probably the limited exposure to these drugs the cause of reduced antimicrobial resistance, a positive correlation that not always is observed. There are no reports on the treatment to eliminate $H$. pylori infection in native Mexican people. Our hypothesis was further strengthened with the observation of absence of resistance to levofloxacin an antibiotic that hardly reach these communities. As mentioned above, the most recent Mexican consensus no longer recommend triple therapy (Bosques-Padilla et al., 2018) based on susceptibility patterns in mestizo populations. Our results suggest triple therapy may still be an effective treatment in native communities and call the attention to differences between populations that must be taken into account when working in consensus for regions with both mestizo and native communities. This is regionally important since most Latin American countries still have indigenous populations (Adhikari et al., 2016).

Bacteria use two major genetic strategies to adapt to the exposure of antibiotics. One is mutations in genes associated with mechanism of action of the drugs and the second is incorporation of resistance gene by acquisition of foreign DNA via horizontal transfer (Munita and Arias, 2016; Tuan et al., 2019). Several reports have identified mutations in the genome of $H$. pylori involved in resistance to clarithromycin, fluoroquinolones, and metronidazole. Sequencing of the $H$. pylori genome is increasingly more available and less expensive and offers a unique opportunity to identify known and novel mutations in genes associated with resistance to antibiotics. Experimentally confirmed mutations can then be identified using even simpler and cheaper techniques, some commercially available and designed for use with biopsy tissues without the need to isolate H. pylori (Poel et al., 2019; Pichon et al., 2020). However, this is only possible after a thorough confirmation of the specific association with resistance in different regions of the world.

H. pylori resistance to clarithromycin is frequently due to point mutation in the $23 \mathrm{~s}$ rRNA gene and prevalence of these mutations varies geographically even between populations in the same country (Kageyama et al., 2019). In Chinese patients, prevalence of A2143G was 10\%-14\% (Savoldi et al., 2018; Zhuoqi et al., 2008) and in Malaysian strains it was $90.5 \%$ (Alfizah et al., 2014). Furthermore, in Iran A2143G was highly frequent in clarithromycin resistant strains (68.7\%) and A2142G was low (5.6\%) (Keshavarz et al., 2015); similarly, A2142G was low (9.5\%) in H. pylori resistant strains from Malaysia (Alfizah et al., 2014), whereas in Brazil A2147G variant was the most common (77.8\%) (Martins et al., 2006). We found good correlation between phenotypic resistant to clarithromycin and mutations in 23s rRNA, mainly because the reported mutations were present in resistant strains and mostly absent in sensitive strains. There were however strains resistant to clarithromycin but without mutations in $23 \mathrm{~s}$ rRNA suggesting the presence of other mechanism of resistance in H. pylori in our population.

Resistance to levofloxacin is given by non-synonymous mutations in a region of gyrA or gyrB, determining resistance to quinolones. Amino acid substitutions in GyrA have been described in positions 91 (D91G, N, A, Y, or H) and 87 (N87L, I, A, or K) (Zerbetto De Palma et al., 2017) in addition to other substitutions in GyrB. 87 and 91 substitutions are the more frequently described, suggesting their presence might predict resistance to fluoroquinolones (Zerbetto De Palma et al., 2017; Lopez Gasca et al., 2018). In Colombia an important increase in fluoroquinolone resistance from 2009 to 2014 was reported, mostly associated with mutations in gyrA, with N87I being the most common variant (Wang et al., 2010; Trespalacios et al., 
2015). In our study, we did not find any substitution in GyrA exclusively present in resistant strains and in fact the performance of molecular detection of mutants was moderate in our population, mainly because of the frequent presence of the described resistant-associated variants in sensitive strains. Limiting the test to the detection of variants in position 87 of GyrA increase concordance but sensitivity is still low (detecting in $77 \%$ of resistant strains). gyrB mutation (S479G) was identified in two $H$. pylori resistant strains, indicating that $g y r$ $B$ has no impact on fluoroquinolone resistance in our population. Our results agree with previous reports from Kuala Lumpur and Iran (Hanafiah et al., 2019; Tuan et al., 2019).

Resistance to metronidazole involves the inactivation of the $r d x A$ gene that catalyzes the reduction of metronidazole (Tuan et al., 2019). A number of studies have reported amino acid variants in RdxA present only in metronidazole resistant strains (Marais et al., 2003; Butlop et al., 2016). In this study we found amino acid variants in $\mathrm{RdxA}$ that were associated exclusively with metronidazole resistant strains in over $50 \%$ of the resistant isolates; however, these variants were as much as 32 and none was present in more than $5 \%$ of resistant isolates. In addition, 32 other variants were present either in both sensitive and resistant strains or only in sensitive isolates. Thus, it is not a surprise the low concordance found with molecular and phenotypic detection; in addition, considering the large number on mutations in the $r d x A$ gene it seems that molecular detection of resistance is not an option for metronidazole. Furthermore, six metronidazole-resistant strains contained stop codons at position 13, 26, 37, 72, 73, 73; different from those reported previously in positions 2, 50, and 52 (Butlop et al., 2016). We found a number of amino acid variants present only in resistant $H$. pylori isolates. Sustitutions in rpsU (A11T) and $\operatorname{dppA}$ (I485L, 37del) were found to be present only in resistant isolates however, the role of these variants in resistance to metronidazole remains to be elucidated because we had a high percentage of resistant strains that did not present any of the studied mutations, whereas other mutations were present in both sensitive and resistant strains. The comparison between the phenotypic and genotypic methods is reported for different authors in various countries and with diverse results (De Francesco et al., 2010; Tuan et al., 2019; Mascellino et al., 2020). Mascellino et al, reported good correlation genotype-phenotype among $H$. pylori resistant to levofloxacin but not in clarithromycin resistant strains (Mascellino et al., 2020). While Tuan et al. (2019) reported excellent genotype to phenotype agreement for clarithromycin and good agreement for levofloxacin and amoxicillin but no agreement for metronidazole.

We have to keep in mind that other mechanisms of metronidazole resistance have been described, including mutations in frxA, dapF, and efflux proteins (Miftahussurur et al., 2016) (Table 8).

In this study, we showed that in Mexican H. pylori strains there was an important increase in resistance to clarithromycin and levofloxacin and appearance of resistant to amoxicillin during a period of 20 years, which has important clinical implications. We also report a significantly lower frequency of antibiotics resistance in $H$. pylori strains from native communities. Finally, we found good to moderate performance of genetic test to detect antibiotic resistance; thus, molecular methods for characterizing resistant genes require further validation in each population.

\section{DATA AVAILABILITY STATEMENT}

The data set analyzed in this work is publicly accessible under the NCI bioproject PRJNA338771 (biosamples: SAMN05569559, SAMN05569561, SAMN05569574, SAMN05569575, SAMN05569581-SAMN05569583, SAMN05569586, SAMN05569587, SAMN05569589SAMN05569592, SAMN11483334 and SAMN11483336); bioproject PRJNA203445 (biosamples: SAMN09935045SAMN09935055, SAMN09935057-SAMN09935061, SAMN09935063, SAMN09935064, SAMN10881935, SAMN10881940, SAMN10881942, SAMN10881945SAMN10881948, SAMN10881953, SAMN10881959, SAMN10881963, SAMN10881965, SAMN10881972) and bioproject PRJNA681870 (biosamples SAMN16970063SAMN16970092 and SAMN16970108-SAMN16970125) (Supplementary Table 1).

\section{ETHICS STATEMENT}

The study was approved by the ethical committee from the Instituto Mexicano del Seguro Social.

\section{AUTHOR CONTRIBUTIONS}

MC-P designed and coordinated the study and wrote the manuscript. JT participated in design and coordination of the study, and wrote the manuscript. AE-O performed the isolation and antimicrobial susceptibility test of $H$. pylori. AG-D participated in statistical analysis. EA-Z and RT participated in the bioinformatics analysis. SG-C contributed with revising the article. All authors contributed to the article and approved the submitted version.

\section{FUNDING}

This work was supported by "Coordinación Nacional de Investigación en Salud, Instituto Mexicano del Seguro Social. México. Grand FIS/IMSS/PROT/G16/1600.

\section{SUPPLEMENTARY MATERIAL}

The Supplementary Material for this article can be found online at: https://www.frontiersin.org/articles/10.3389/fcimb.2020. 539115/full\#supplementary-material 


\section{REFERENCES}

Adhikari, K., Mendoza-Revilla, J., Chacón-Duque, J. C., Fuentes-Guajardo, M., and Ruiz-Linares, A. (2016). Admixture in Latin America. Curr. Opin. Genet. Dev. 41, 106-114. doi: 10.1016/j.gde.2016.09.003

Alarcón-Millán, J., Fernández-Tilapa, G., Cortés-Malagón, E. M., Castañón-Sánchez, C. A., De Sampedro-Reyes, J., Cruz-del Carmen, I., et al. (2016). Clarithromycin resistance and prevalence of Helicobacter pylori virulent genotypes in patients from Southern México with chronic gastritis. Infect. Genet. Evol. 44, 190-198. doi: 10.1016/j.meegid.2016.06.044

Alba, C., Blanco, A., and Alarcón, T. (2017). Antibiotic resistance in Helicobacter pylori. Curr. Opin. Infect. Dis. 30, 489-497. doi: 10.1097/ QCO.0000000000000396

Alfizah, H., Norazah, A., Hamizah, R., and Ramelah, M. (2014). Resistotype of Helicobacter pylori isolates: The impact on eradication outcome. J. Med. Microbiol. 63, 703-709. doi: 10.1099/jmm.0.069781-0

Avilés-Jiménez, F., Reyes-Leon, A., Nieto-Patlán, E., Hansen, L., Burgueño, J., Ramos, I., et al. (2012). In Vivo Expression of Helicobacter pylori Virulence Genes in Patients With Gastritis, Ulcer, and Gastric Cancer. Infect. Immun. 80, 594-601. doi: 10.1128/IAI.05845-11

Bosques-Padilla, F. J., Remes-Troche, J. M., González-Huezo, M. S., Pérez-Pérez, G., Torres-López, J., Abdo-Francis, J. M., et al. (2018). IV consenso mexicano sobre Helicobacter pylori. Rev. Gastroenterol. México 83, 325-341. doi: 10.1016/ j.rgmx.2018.05.003

Butlop, T. R. P., Mungkote, N. T. N., and Chaichanawongsaroj, N. T. R. (2016). Analysis of allelic variants of $\mathrm{rdxA}$ associated with metronidazole resistance in Helicobacter pylori: detection of common genotypes in rdxA by multiplex allele-specific polymerase chain reaction. Genet. Mol. Res. 15, 1-11. doi: 10.4238/gmr.15038674

Camargo, M. C., García, A., Riquelme, A., Camargo, C. A., Hernandez-garcía, T., Candia, R., et al. (2015). The problem of Helicobacter pylori resistance to antibiotics: a systematic Review in Latin America. Am. J. Gastroenterol. 2014, 485-495. doi: 10.1038/ajg.2014.24.The

Camorlinga-Ponce, M., Perez-Perez, G., Gonzalez-Valencia, G., Mendoza, I., Peñaloza-Espinosa, R., Ramos, I., et al. (2011). Helicobacter pylori genotyping from american indigenous groups shows novel amerindian vacA and cagA alleles and Asian, African and European admixture. PloS One 6 (11), e 27212. doi: 10.1371/journal.pone.0027212

Carver, T., Harris, S. R., Berriman, M., Parkhill, J., and McQuillan, J. A. (2012). Artemis: An integrated platform for visualization and analysis of highthroughput sequence-based experimental data. Bioinformatics 28, 464-469. doi: 10.1093/bioinformatics/btr703

Curado, M., Moura de Oliveira, M., and Araujo, M. (2019). Prevalence of Helicobacter Pylori Infection in Latin America and the Caribbean Populations: A Systematic Review and Meta-Analysis. Cancer Epidemiol. 60, 141-148. doi: 10.1016/j.canep.2019.04.003

Dadgostar, P. (2019). Antimicrobial resistance: implications and costs. Infect. Drug Resist. 12, 3903-3910. doi: 10.2147/IDR.S234610

Dang, B., and Graham, D. (2017). Helicobacter pylori Infection and Antibiotic Resistance: A WHO High Priority? Nat. Rev. Gastroenterol. Hepatol. 14, 383384. doi: 10.1038/nrgastro.2017.57

De Francesco, V., Zullo, A., Ierardi, E., Giorgio, F., Perna, F., Hassan, C., et al. (2010). Phenotipic and genotypic Helicobacter pylori clarithromycin resistance and therapeutic outcome: benefits and limits. J. Antimicrob. Chemother. 65 (2), 327-332. doi: 10.1093/jac/dkp445

Draper, J. L., Hansen, L. M., Bernick, D., Abedrabbo, S., Underwood, J. G., Kong, N., et al. (2017). Fallacy of the Unique Genome: Sequence Diversity within Single Helicobacter pylori Strains. mBio 8 (1), e02321-16. doi: 10.1128/ mBio.02321-16

Edgar, R. C. (2004). MUSCLE: Multiple sequence alignment with high accuracy and high throughput. Nucleic Acids Res. 32, 1792-1797. doi: 10.1093/ nar/gkh340

Farzi, N., Yadegar, A., Sadedhi, A., Asadzadeh, A., Marian, S., Raymond, J., et al. (2019). High Prevalence of Antibiotic Resistance in Iranian Helicobacter pylori Isolates: Importance of Functional and Mutational Analysis of Resistance Genes and Virulence Genotyping. J. Clin. Med. 8 (11), 1-18. doi: 10.3390/ jcm 8112004
Fiorini, G., Zullo, A., Saracino, I. M., Gatta, L., Pavoni, M., and Vaira, D. (2018). Pylera and sequential therapy for first-line Helicobacter pylori eradication. Eur. J. Gastroenterol. Hepatol. 30, 621-625. doi: 10.1097/ MEG.0000000000001102

Fuccio, L., Henry Eusebi, L., Maurizio Zagari, R., and Bazzoli, F. (2009). Helicobacter pylori Eradication Treatment Reduces but Does Not Abolish the Risk of Gastric Cancer. Am. J. Gastroenterol. 104, 3100-3101. doi: 10.1038/ ajg.2009.516

Goderska, K., Agudo Pena, S., and Alarcon, T. (2018). Helicobacter pylori treatment: antibiotics or probiotics. Appl. Microbiol. Biotechnol. 102, 1-7. doi: 10.1007/s00253-017-8535-7

Gonzalez-Hormazabal, P., Musleh, M., Escandar, S., Valladares, H., Lanzarini, E., Castro, V., et al. (2018). Prevalence of Clarithromycin Resistance in Helicobacter pylori in Santiago, Chile, Estimated by Real-Time PCR Directly From Gastric Mucosa. BMC. Gastroenterol. 18, 91-91. doi: 10.1186/s12876018-0820-0

Goodwin, A., Kersulyte, D., Sisson, G., Veldhuyzen van Zanten, S. J., Berg, D. E., and Hoffman, P. S. (1998). Metronidazole resistance in Helicobacter pylori is due to null mutations in a gene ( $\mathrm{rdxA}$ ) that encodes an oxygen-insensitive NADPH nitroreductase. Mol. Microbiol. 28, 383-393. doi: 10.1046/j.13652958.1998.00806.x

Guzman-Rosas, S. (2016). La interculturalidad en salud: espacio de convergencia entre dos sistemas de conocimiento*. Rev. Gerenc. Polit. Salud. 15, 10-29. doi: 10.11144/Javeriana.rgyps15-31.isec

Hanafiah, A., Binmaeil, H., Raja, R., Mohamed, I., and Lopes, B. (2019). Molecular Characterization and Prevalence of Antibiotic Resistance in Helicobacter pylori Isolates in Kuala Lumpur, Malaysia. Infect. Drug Resistant 12, 3051-3061. doi: 10.2147/IDR.S219069

Hashemi, S., Farajzadeh, A., Goodarzi, H., Jaafar, M., Saeid, S., Aslani, S., et al. (2019). Genetic basis for metronidazole and clarithromycin resistance in Helicobacter pylori strains isolated from patients with gastroduodenal disorders. Infect. Drug Resistant 12, 535-543. doi: 10.3390/jcm 9061930

Heo, J., and Jeon, S. W. (2014). Optimal treatment strategy for Helicobacter pylori: era of antibiotic resistance. World J. Gastroenterol. 20, 5654-5659. doi: 10.3748/wjg.v20.i19.5654

Hooi, J., Ying, W., Khoon, W., Suen, M., Underwood, F., Tanyingoh, D., et al. (2017). Global Prevalence of Helicobacter pylori Infection: Systematic Review and Meta-Analysis. Gastroenterology 153, 420-429. doi: 10.1053/j.gastro. 2017.04.022

Ierardi, E., Giorgio, F., Losurdo, G., Di Leo, A., and Principi, M. (2013). How antibiotic resistances could change Helicobacter pylori treatment: A matter of geography? World J. Gastroenterol. 19, 8168-8180. doi: 10.3748/ wjg.v19.i45.8168

Kageyama, C., Sato, M., Sakae, H., Obayashi, Y., Kawahara, Y., Mima, T., et al. (2019). Increase in antibiotic resistant Helicobacter pylori in a University Hospital in Japan. Infect. Drug Resist. 12, 597-602. doi: 10.2147/IDR.S196452

Keshavarz, A., Moniri, R., Saffari, M., Razavi Zadeh, M., Arj, A., Mousavi, S. G. A., et al. (2015). The Helicobacter pylori Resistance Rate to Clarithromycin in Iran. Microb. Drug Resist. 21, 69-73. doi: 10.1089/mdr.2014.0104

Kong, N., Thao, K., Ng, W., Agulto, R., Weiss, A., Spittle, K., et al. (2014). Automation of PacBio SMRTbell $10 \mathrm{~kb}$ Template Preparation on an Agilent NGS Workstation. Agil. Technol. doi: 10.13140/RG.2.1.4403.2725

Ladrón-de-Guevara, L., Bornstein-Quevedo, L., González-Huezo, S., CastañedaRomero, B., Costa, F. G., and di Silvio-López, M. (2019). Helicobacter pylori eradication in Mexico with a levofloxacin-based scheme versus standard triple therapy: Results from an open-label, randomized, noninferiority phase iiib trial. Rev. Gastroenterol. Mex. 84, 274-283. doi: 10.1016/j.rgmx.2018.04.005

Laxminarayan, R., Duse, A., Wattal, C., Zaidi, A. K. M., Wertheim, H. F. L., Sumpradit, N., et al. (2013). Antibiotic resistance - the need for global solutions. Lancet Infect. Dis. 13, 1057-1098. doi: 10.1016/S1473-3099(13) 70318-9

Leja, M., Axon, A., and Brenner, H. (2016). Epidemiology of Helicobacter pylori infection. Helicobacter 21, 3-7. doi: 10.1111/hel.12332

Liou, J.-M., Bair, M.-J., Chen, C.-C., Lee, Y.-C., Chen, M.-J., Chen, C.-C., et al. (2016). Levofloxacin Sequential Therapy vs Levofloxacin Triple Therapy in the Second-Line Treatment of Helicobacter pylori: A Randomized Trial. Am. J. Gastroenterol. 111, 381-387. doi: 10.1038/ajg.2015.439 
Lopez Gasca, M., Peña, J., Garcia Amado, M., Michelangeli, F., and Contreras, M. (2018). Point Mutations at gyrA and gyrB Genes of Levofloxacin-Resistant Helicobacter pylori Isolates in the Esophageal Mucosa From a Venezuelan Population. Am. J. Trop. Med. Hyg. 98, 1051-1055. doi: 10.4269/ajtmh.17-0478

Malfertheiner, P., Megraud, F., O’Morain, C. A., Gisbert, J. P., Kuipers, E. J., Axon, A. T., et al. (2017). Management of Helicobacter pylori infection-the Maastricht V/Florence consensus report. Gut 66, 6-30. doi: 10.1136/gutjnl2016-312288

Marais, A., Bilardi, C., Cantet, F., Mendz, G. L., and Mégraud, F. (2003). Characterization of the genes $\operatorname{rdxA}$ and frxA involved in metronidazole resistance in Helicobacter pylori. Res. Microbiol. 154, 137-144. doi: 10.1016/ S0923-2508(03)00030-5

Marques, A. T., Vitor, J., Santos, A., Oleastro, M., and Vale, F. (2020). Trends in Helicobacter pylori resistance to clarithomycin: from phenotypic to genomic approaches. Microbial. Genomics 6, 1-11. doi: 10.1099/mgen.0.000344

Martins, L. C., de Oliveira Corvelo, T. C., Oti, H. T., do Socorro Pompeu Loiola, R., Aguiar, D. C. F., dos Santos Barile, K. A., et al. (2006). ABH and Lewis antigen distributions in blood, saliva and gastric and $H$ pylori infection in gastric ulcer patients. World J. Gastroenterol. 12, 1120-1124. doi: 10.3748/wjg.v12.i7.1120

Mascellino, M., Oliva, A., Miele, M., De Angelis, M., and Bruno, G. (2020). Secondary antibiotic resistance, correlation gentypic and phenotypic methods and treatment in Helicobacter pylori infected patients: A retrospective study. Antibiotics 9, 549-559. doi: 10.3390/antibiotics9090549

Megraud, F., Coenen, S., Versporten, A., Kist, M., Lopez-Brea, M., Hirschl, A. M., et al. (2013). Helicobacter pylori resistance to antibiotics in Europe and its relationship to antibiotic consumption. Gut 62, 34-42. doi: 10.1136/gutjnl2012-302254

Mera, R., Fontham, E. T. H., Bravo, L. E., Bravo, J. C., Piazuelo, M. B., Camargo, M. C., et al. (2005). Long term follow up of patients treated for Helicobacter pylori infection. Gut 54, 1536-1540. doi: 10.1136/gut.2005.072009

Miftahussurur, M., Syam, A. F., Nusi, I. A., Makmun, D., Waskito, L. A., Zein, L. H., et al. (2016). Surveillance of Helicobacter pylori antibiotic susceptibility in Indonesia: Different resistance types among regions and with novel genetic mutations. PloS One 11, 1-17. doi: 10.1371/journal.pone.0166199

Miftahussurur, M., Fauzia, K. A., Nusi, I. A., Setiawan, P. B., Syam, A. F., Waskito, L. A., et al. (2020). E-test versus agar dilution for antibiotic susceptibility testing of Helicobacter pylori: a comparison study. BMC Res. Notes 13, 9. doi: 10.1186/ s13104-019-4877-9

Moodley, Y., Linz, B., Bond, R. P., Nieuwoudt, M., Soodyall, H., Schlebusch, C. M., et al. (2012). Age of the association between Helicobacter pylori and man. PloS Pathog. 8, e1002693. doi: 10.1371/journal.ppat.1002693

Moss, S. F. (2017). The Clinical Evidence Linking Helicobacter pylori to Gastric Cancer. Cell Mol. Gastroenterol. Hepatol. 3, 183-191. doi: 10.1016/ j.jcmgh.2016.12.001

Munita, J. M., and Arias, C. A. (2016). Mechanisms of Antibiotic Resistance. Microbiol. Spectr. 4, 1-37. doi: 10.1128/microbiolspec.VMBF-0016-2015

Muñoz-Ramírez, Z. Y., Mendez-Tenorio, A., Kato, I., Bravo, M. M., Rizzato, C., Thorell, K., et al. (2017). Whole genome sequence and phylogenetic analysis show Helicobacter pylori strains from Latin America have followed a unique evolution pathway. Front. Cell. Infect. Microbiol. 7, 50. doi: 10.3389/ fcimb.2017.00050

Nishizawa, T., and Suzuki, H. (2014). Mechanisms of Helicobacter pylori antibiotic resistance and molecular testing. Front. Mol. Biosci. 1, 19. doi: 10.3389/ fmolb.2014.00019

Ogata, S. K., Godoy, A. P. O., da Silva Patricio, F. R., and Kawakami, E. (2013). High Helicobacter pylori resistance to metronidazole and clarithromycin in Brazilian children and adolescents. J. Pediatr. Gastroenterol. Nutr. 56, 645-648. doi: 10.1097/MPG.0b013e31828b3669

O'Connor, A., Gisbert, J. P., O'Morain, C., and Ladas, S. (2015). Treatment of Helicobacter pylori Infection 2015. Helicobacter 20 Suppl 1, 54-61. doi: 10.1111/hel.12258

Page, A. J., Taylor, B., Delaney, A. J., Soares, J., Seemann, T., Keane, J. A., et al. (2016). SNP-sites: rapid efficient extraction of SNPs from multi-FASTA alignments. Microb. Genomics 21-5 (4), e000056. doi: 10.1099/mgen.0.000056

Park, J. Y., Dunbar, K. B., Mitui, M., Arnold, C. A., Lam-Himlin, D. M., Valasek, M. A., et al. (2016). Helicobacter pylori Clarithromycin Resistance and Treatment Failure Are Common in the USA. Dig. Dis. Sci. 61, 2373-2380. doi: 10.1007/s10620-016-4091-8
Pichon, M., Pichard, B., Barrioz, T., Plouzenau, C., Croquet, V., Fotsing, G., et al. (2020). Diagnostic Accuracy of a Non-Invasive Test for the Detection of Helicobacter pylori and Resistance to Clarithromycin in Stool by Real-Time PCR Amplidiag ${ }^{\circledR}$ H. pylori + ClariR Assay. J. Clin. Microbiol. 58 (4), e0178719. doi: 10.1128/JCM.01787-19

Poel, B., Gils, S., Micalessi, I., Carton, S., Christiaens, P., Cuyle, P. J., et al. (2019). Molecular Detection of Helicobacter pylori and Clarithromycin Resistance in Gastric Biopsies: A Prospective Evaluation of RIDA ${ }^{\circledR}$ GENE Helicobacter pylori Assay. Acta Clin. Belg. 17, 1-7. doi: 10.1080/17843286.2019.1685741

Rimbara, E., Noguchi, N., Kijima, H., Yamaguchi, T., Kawai, T., and Sasatsu, M. (2007). Mutations in the 23S rRNA gene of clrarithromycin resistant Helicobacter pylori from Japan. Int. J. Antimicro Agents 30 (3), 250-254. doi: 10.1016/j.ijantimicag.2007.04.009

Rollan, A., Cortés, P., Durán, L., Pablo Arab, J., Candia, R., Espino, A., et al. (2014). Clinical trials study Management of Helicobacter pylori infection in Latin America: A Delphi technique-based consensus. World J. Gastroenterol. 20, 10969-10983. doi: 10.3748/wjg.v20.i31.10969

Romero-Hidalgo, S., Ochoa-Leyva, A., Garcíarrubio, A., Acuña-Alonzo, V., Antúnez-Argüelles, E., Balcazar-Quintero, M., et al. (2017). Demographic history and biologically relevant genetic variation of Native Mexicans inferred from whole-genome sequencing. Nat. Commun. 8, 1005. doi: 10.1038/s41467-017-01194-Z

Saranathan, R., Levi, M., Wattan, A., Malek, A., Asare, E., Behin, D., et al. (2020). Helicobacter pylori infectious in the Bronx, New York: Surveying antibiotic susceptibility and strain lineage by whole-genome sequencing. J. Clin. Microbiol. 58 (3), 1-13. doi: 10.1128/JCM.01591-19

Savoldi, A., Carrara, E., Graham, D. Y., Conti, M., and Tacconelli, E. (2018). Prevalence of antibiotic resistance in Helicobacter pylori: A systematic Review and metaanalysis in World Health Organization Regions. Gastroenterology 155 (5), 1372-1382. doi: 10.1053/j.gastro.2018.07.007

Seemann, T. (2014). Prokka: rapid prokaryotic genome annotation. Bioinformatics 30, 2068-2069. doi: 10.1093/bioinformatics/btu153

Sheu, B.-S., Wu, M.-S., Chiu, C.-T., Lo, J.-C., Wu, D.-C., Liou, J.-M., et al. (2017). Consensus on the clinical management, screening-to-treat, and surveillance of Helicobacter pylori infection to improve gastric cancer control on a nationwide scale. Helicobacter 22, 1-15. doi: 10.1111/hel.12368

Simpson, J. T., Wong, K., Jackman, S. D., Shein, J. E., Jones, S. J., and Birol, 1. (2009). ABySS: A parallel assembler for short read sequence data ABySS : A parallel assembler for short read sequence data. Genome Res. 19, 1117-1123. doi: 10.1101/gr.089532.108

Sjomina, O., Pavlova, J., Niv, Y., and Leja, M. (2018). Epidemiology of Helicobacter pylori infection. Helicobacter 23 suppl 1, 1-15. e12514. doi: 10.1111/hel.12514

Smith, S. M., O'Morain, C., and McNamara, D. (2014). Antimicrobial susceptibility testing for Helicobacter pylori in times of increasing antibiotic resistance. World J. Gastroenterol. 20, 9912-9921. doi: 10.3748/ wjg.v20.i29.9912

Suzuki, S., Esaki, M., Kusano, C., Ikehara, H., and Gotoda, T. (2019). Development of Helicobacter pylori treatment: How do we manage antimicrobial resistance? World J. Gastroenterol. 25, 1907-1912. doi: 10.3748/wjg.v25.i16.1907

Tamura, K., Stecher, G., Peterson, D., Filipski, A., and Kumar, S. (2013). MEGA6: Molecular Evolutionary Genetics Analysis version 6.0. Mol. Biol. Evol. 30, 2725-2729. doi: 10.1093/molbev/mst197

Thung, I., Aramin, H., Vavinskaya, V., Gupta, S., Park, J. Y., Crowe, S. E., et al. (2016). Review article: the global emergence of Helicobacter pylori antibiotic resistance. Aliment. Pharmacol. Ther. 43, 514-533. doi: 10.1111/apt.13497

Torres, J., Camorlinga-Ponce, M., Pérez-Përez, G., Madrazo-De la Garza, A., Dehesa, M., González-Valencia, G., et al. (2001). Increasing multidrug resistance in Helicobacter pylori strains isolated from children and adults in Mexico. J. Clin. Microbiol. 39, 2677-2680. doi: 10.1128/JCM.39.7.26772680.2001

Trespalacios, A. A., Rimbara, E., Otero, W., Reddy, R., and Graham, D. Y. (2015). Improved allele-specific PCR assays for detection of clarithromycin and fluoroquinolone resistant of Helicobacter pylori in gastric biopsies: identification of N87I mutation in GyrA. Diagn. Microbiol. Infect. Dis. 81, 251-255. doi: 10.1016/j.diagmicrobio.2014.12.003

Tshibangu-Kabamba, E., De jesus, P., Phuoc, V., Matzumoto, T., Akada, J., Kido, Y., et al. (2020). Next generation sequencing of the whole bacterial genome for tracking molecular insight into the broad spectrum antimicrobial resistance of 
Helicobacter pylori clinical isolates from the Democractic Republic of Congo. Microorganisms 8, 1-16. doi: 10.3390/microorganisms 8060887

Tuan, V. P., Narith, D., Tshibangu-kabamba, E., Dang, H., and Dung, Q. (2019). A Next-Generation Sequencing-Based Approach to Identify Genetic Determinants of Antibiotic Resistance in Cambodian Helicobacter pylori Clinical Isolates J. Clin. Med. 8 (6), 858. doi: $10.3390 / \mathrm{jcm} 8060858$

Vázquez Tsuji Óscar, C. R. T. (2010). Regulación de la venta de antibióticos en México. Rev. Enfer. Infect. Pediatr. XXIII, 47.

Wang, L., Cheng, H., Hu, F., and Li, J. (2010). Distribution of gyrA mutations in fluoroquinolone-resistant Helicobacter pylori strains World J. Gastroenterol. 16, 2272-2277. doi: 10.3748/wjg.v16.i18.2272

Weimer, B. C. (2017). 100K Pathogen Genome Project. Genome Announc. 5 (28), 3-4. doi: 10.1128/genomea.00594-17

Weis, A. M., Storey, D. B., Taff, C. C., Townsend, A. K., Huang, B. C., Kong, N. T., et al. (2016). Genomic Comparison of Campylobacter spp. and Their Potential for Zoonotic Transmission between Birds, Primates, and Livestock. Appl. Environ. Microbiol. 82 (24), 7165-7175. doi: 10.1128/aem.01746-16

Wu, T., Chuah, S., Lee, C. H., Liang, C. M., Lu, L., Kuo, Y. H., et al. (2015). Five-year Sequential Changes in Secondary Antibiotic Resistance of Helicobacter pylori in Taiwan. World J. Gastroenterol. 21, 106669-106674. doi: 10.3748/wjg.v21.i37.10669

Zerbetto De Palma, G., Mendiondo, G., Wonaga, A., and Viola, L. (2017). Occurrence of Mutations in the Antimicrobial Target Genes Related to
Levofloxacin, Clarithromycin, and Amoxicillin Resistance in Helicobacter pylori Isolates from Buenos Aires C. Microb. Drug Resist. 23, 351-358. doi: 10.1089/mdr.2015.0361

Zerbino, D. R., and Birney, E. (2008). Velvet: Algorithms for de novo short read assembly using de Bruijn graphs. Genome Res. 18 (5), 821-882. doi: 10.1101/ gr.074492.107

Zhuoqi, L., Shen, J., Zhang, L., Shen, L., Baozhen, Q., Zhou, J., et al. (2008). Prevalence of A2143G mutation of $\mathrm{H}$. pylori-23S rRNA in Chinese subjects with and without clarithromycin use history. BMC Microbiol. 8, 1-8. doi: $10.1186 / 1471-2180-8-81$

Conflict of Interest: The authors declare that the research was conducted in the absence of any commercial or financial relationships that could be construed as a potential conflict of interest.

Copyright (c) 2021 Camorlinga-Ponce, Gómez-Delgado, Aguilar-Zamora, Torres, Giono-Cerezo, Escobar-Ogaz and Torres. This is an open-access article distributed under the terms of the Creative Commons Attribution License (CC BY). The use, distribution or reproduction in other forums is permitted, provided the original author (s) and the copyright owner(s) are credited and that the original publication in this journal is cited, in accordance with accepted academic practice. No use, distribution or reproduction is permitted which does not comply with these terms. 